\title{
Simulation of Unsaturation Domain Formation in Antiferromagnetic Multilayers
}

\author{
M. Major, L. Bottyán AND D.L. NAGY \\ KFKI Research Institute for Particle and Nuclear Physics \\ P.O.B. 49, 1525 Budapest, Hungary
}

(Received November 19, 2001)

\begin{abstract}
A two-dimensional phenomenological model of patch-domain formation on unsaturation in strongly coupled tri- and multilayer antiferromagnets is presented. Domain patterns are simulated using a fast Monte Carlo algorithm. As a first example the autocorrelation function of the simulated domain pattern is compared with that of a measured Kerr image.
\end{abstract}

PACS numbers: 75.70.Kw, 75.40.Mg, 78.20.Ls, 75.25.+z

\section{Introduction}

Magnetic multilayers (ML) have been for a long time in the focus of interest due to their novel material properties and diverse technological applications. The antiferromagnetically (AF) coupled $\mathrm{Fe} / \mathrm{Cr}$ system, for example, shows the giant magnetoresistance (GMR) effect [1]. The GMR noise can be influenced by the domain structure of the ML [2].

Recently on an AF-coupled Fe/Cr ML with an in-plane fourfold anisotropy a domain size tailoring mechanism was found by off-specular synchrotron Mössbauer reflectometry (SMR) and verified by polarized neutron reflectometry (PNR) [3]. Small ( $\mu \mathrm{m}$ size) domains are formed when the external magnetic field $H_{\text {ext }}$ is decreased from the saturation region. Most of these domains are replaced by at least one order of magnitude bigger ones (domain coarsening) on passing the spin-flop transition [4-6]. SMR and PNR intensities can be calculated from the lateral autocorrelation function of the AF domains which, in turn, is determined by the AF domain pattern. A simple phenomenological model and Monte Carlo simulations 
of domain patterns are presented here reproducing the domain nucleation on "unsaturation" i.e. on leaving the saturation region in decreasing magnetic field. The same algorithm with similar "flipping rules" was applied to simulate the domain coarsening observed at the spin-flop process [7]. Autocorrelation functions of model and experimental patch-domain patterns are adjusted.

\section{Unsaturation patch domains}

In a strongly AF-coupled metallic ML the domain structure of the individual ferromagnetic layers is rather strictly correlated through the ML stack from the substrate to the surface allowing for a two-dimensional representation of the domains.

The term "strongly coupled" refers to the fact that the anisotropy energies are at least one order of magnitude smaller than the coupling energies. In such systems the unsaturation domain formation is governed by the AF coupling, it involves coherent rotation of spins and results in a characteristic patch-domain pattern [8].

The domain formation is associated with the lateral correlation length of the saturation field variation of $H_{\text {sat }}$ [3], related to the unavoidable variation of the thickness of $\mathrm{Cr}$ spacer. As a consequence, a region will have the freedom to decide the sense of rotation of the top layer when the decreasing $H_{\text {ext }}$ reaches the local value of $H_{\text {sat }}$ [3]. Neighbour regions within the correlation length of $H_{\text {sat }}$ will choose the same sense of rotation, to avoid creating new domain walls [3]. Here we show that this simple ansatz is enough to explain the observed [8] patch-like shape of the domains and that even the autocorrelation function of the observed domains can be reproduced with a surprisingly high accuracy.

\section{Modelling the patch domains}

The ML is modelled by a two-dimensional matrix of pixels where the "colour" (black or white) of each pixel represents the direction of the magnetization (of the topmost layer in the ML) in a given pixel area. The mesh size of the grid is smaller than the actually formed domains (see below) but still "classical", i.e., consists of many columns of atoms. (In the particular case of the presented simulation for example 1 pixel $=146 \times 146 \mathrm{~nm}^{2}$.)

The domains are continuous regions of the same colour on the grid. Domain formation is governed by the distribution of $H_{\text {sat }}[3]$. The higher the saturation field of a given pixel is, the sooner (i.e. at higher $H_{\text {ext }}$ ) the pixel unsaturates. To obtain the saturation field distribution $H_{\text {sat }}(r)$, we take a grid of uncorrelated random numbers $\{U(r)\}$ of Gaussian distribution [9], where $r=(x, y)$ is the position vector, then smooth $\{U(r)\}$ with an empirical function of smoothing width $w$ : 


$$
H_{\mathrm{sat}}(\boldsymbol{r})=\sum_{\left|\boldsymbol{r}-\boldsymbol{r}^{\prime}\right|<w}\left(1-\frac{\left(\boldsymbol{r}-\boldsymbol{r}^{\prime}\right)^{2}}{w^{2}}\right) U\left(\boldsymbol{r}^{\prime}\right) .
$$

Periodic boundary conditions are used throughout [7].

Unsaturation is modelled in the following. Above $\max \left\{H_{\text {sat }}(r)\right\}$ all pixels are in saturation (represented by grey), then $H_{\text {ext }}$ is lowered. When $H_{\text {ext }}$ matches $H_{\text {sat }}(\boldsymbol{r})$ of a given pixel at $r$, the pixel unsaturates, i.e., chooses its sense of rotation (black (ML top layer): left, white (ML top layer): right). Creation of excess domain walls may be avoided by obeying the so-called "flipping rules" as follows. (A) The pixel chooses the colour of the surrounding majority (all already flipped first neighbours (maximum 8) count with equal weight, pixels still in saturation having no influence) and (B) the pixel chooses colour by random if no decision can be made according to the previous rule. At $H_{\text {ext }}=\min \left\{H_{\text {sat }}(r)\right\}$ the ML is completely unsaturated. In Fig. 1a the $50 \%$ unsaturated state, while in Fig. $1 \mathrm{~b}$ the totally unsaturated state can be seen.

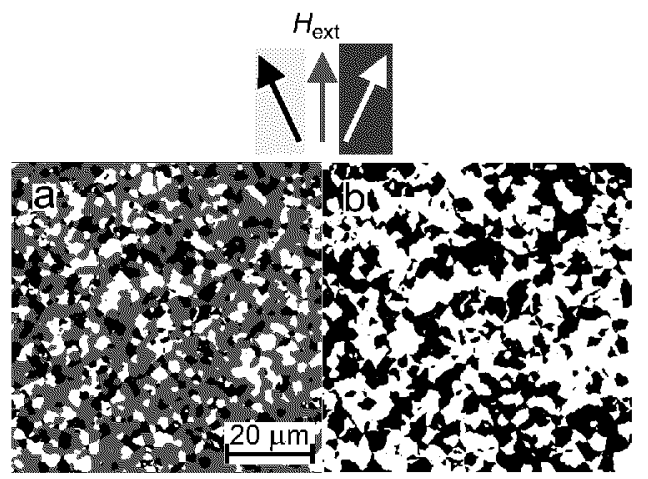

Fig. 1. Unsaturation domain nucleation. In (a) half of the pixels is still in saturation (grey). In (b) the completely unsaturated image is shown. Black pixels represent layer stacks with the top layer more to the left, while white pixels more to the right. The external field is applied along the grey arrow. The length scale corresponds to the fit to the Kerr microscopic image [8]. The full grid is 500 by 500 pixels, consequently 1 pixel corresponds to $146 \times 146 \mathrm{~nm}^{2}$.

A direct realization of the above algorithm leads to rather time-consuming calculations. Indeed, to find the next pixel in the grid to unsaturate when decreasing $H_{\text {ext }}$, all pixels have to be ordered according to their value of $H_{\text {sat }}$. This can be, however, circumvented by a fast algorithm in the following way. The grid is scanned for grey pixels. When found it is checked if all grey neighbours possess a lower $H_{\text {sat }}$ value than the one found. If yes, the pixel is allowed to choose its colour according to the above flipping rules. If not, the next pixel is chosen. The scan of the grid is repeated as long as all grey pixels flip to either black or white. The final state generated by this "greyscan" algorithm is easily seen to be equivalent with 
that of the algorithm of ordered pixels. The subsequent greyscans, admittedly, do not represent a temporal evolution of the domain shapes. This latter, however, can be reproduced and a "movie" of the domain formation constructed from the final state by varying $H_{\text {ext }}$ and flipping the pixel colour back to grey for $H_{\text {sat }}(\boldsymbol{r})<H_{\text {ext }}$.

Let us note that the above model applies to both trilayers and MLs as far as the two-dimensional representation is valid. Furthermore, patch domains are formed close to saturation, not depending on in-plane anisotropy and biquadratic coupling if the bilinear coupling is strong as compared to the other energy terms, resulting in coherent domain rotation in fields decreasing down to zero.

\section{Comparison with experimental results}

In order to derive measurable quantities from the simulation the autocorrelation function $C(r)$ of the in-plane direction of the magnetization (and consequently that of the domain image) may be invoked as

$$
C(\boldsymbol{r})=\frac{\sum_{\boldsymbol{r}^{\prime}}\left[m\left(\boldsymbol{r}^{\prime}\right)-\bar{m}\right]\left[m\left(\boldsymbol{r}^{\prime}+\boldsymbol{r}\right)-\bar{m}\right]}{\sum_{\boldsymbol{r}^{\prime}}\left[m\left(\boldsymbol{r}^{\prime}\right)-\bar{m}\right]^{2}},
$$

where $m(\boldsymbol{r})$ represents the chosen "unsaturation" direction (colour) of the given pixel (white: +1 , black: -1$), \bar{m}=\left(1 / L^{2}\right) \sum_{r} m(\boldsymbol{r})$ is the average of $m(\boldsymbol{r})$ and $L$ is the width of the image in pixels. The sum runs over the complete grid.

In order to test our model, the autocorrelation function of the simulated domains and that of published Kerr images [8] were compared and adjusted (Fig. 2). The agreement is quite remarkable for the mesh size of $146 \mathrm{~nm}$ and $w=10$ pixels.

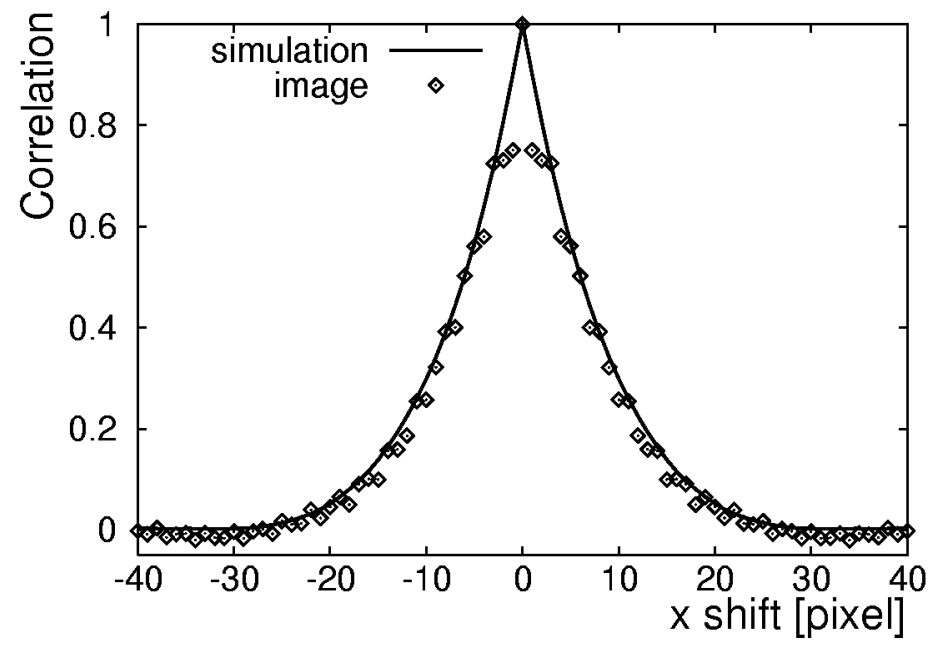

Fig. 2. The autocorrelation function (2) of the Kerr microscopic image [8] as compared with the autocorrelation of the simulated image in Fig. $1 \mathrm{~b}$ with $w=10$ and pixel size of $146 \times 146 \mathrm{~nm}^{2}$. 
Let us note that for a given correlation length of $H_{\text {sat }}(\boldsymbol{r})$ the domain correlation length for an ML of $n$ ferromagnetic layers will scale as $1 / \sqrt{n-1}$. Indeed, each space layer mediating the AF coupling will have the same surface density of domain nucleation centres (local maxima of $H_{\text {sat }}(r)$ ). Consequently, the surface density of domain nucleation centres for the whole ML scales with $(n-1)$ leading to a domain correlation length proportional to $1 / \sqrt{n-1}$. In the first Born approximation the off-specularly scattered intensity is proportional to the Fourier transform of $C(\boldsymbol{r})$. Comparison of $C(\boldsymbol{r})$ of model images with the off-specularly scattered SMR and PNR intensity profiles is in progress.

\section{Conclusions}

A Monte Carlo simulation of patch-domain formation in AF MLs leaving the saturation field region was presented. The generated patch-domain pattern is in quantitative agreement with published Kerr microscopic images of a $\mathrm{Fe} / \mathrm{Cr} / \mathrm{Fe}$ trilayer. The autocorrelation function deduced from the pattern is, in principle, suitable to describe off-specular SMR and PNR measurements.

\section{Acknowledgments}

Support by the project No. T 029409 of the Hungarian Scientific Research Fund (OTKA) and by the Hungarian Academy of Sciences, contract No. 2000-103 2,3 is gratefully acknowledged.

\section{References}

[1] M.N. Baibich, J.M. Broto, A. Fert, F. Nguyen Van Dau, F. Petroff, Phys. Rev. Lett. 61, 2472 (1988).

[2] H.T. Hardner, M.B. Weissman, S.S.P. Parkin, Appl. Phys. Lett. 67, 1938 (1995).

[3] D.L. Nagy, L. Bottyán, B. Croonenborghs, L. Deák, B. Degroote, J. Dekoster, H.J. Lauter, V. Lauter-Pasyuk, O. Leupold, M. Major, J. Meersschaut, O. Nikonov, A. Petrenko, R. Rüffer, H. Spiering, E. Szilágyi, submitted to Phys. Rev. Lett.

[4] B. Dieny, J.P. Gavigan, J.P. Rebouillat, J. Phys. C 2, 159 (1990).

[5] K. Temst, E. Kunnen, V.V. Moshchalkov, H. Maletta, H. Fritzsche, Y. Bruynseraede, Physica B 276-278, 684 (2000).

[6] L. Bottyán, L. Deák, J. Dekoster, E. Kunnen, G. Langouche, J. Meersschaut, M. Major, D.L. Nagy, H.D. Rüter, E. Szilágyi, K. Temst, J. Magn. Magn. Mater., in press.

[7] M. Major, L. Bottyán, D.L. Nagy, J. Magn. Magn. Mater., in press.

[8] M. Rührig, R. Schäfer, A. Hubert, R. Mosler, J.A. Wolf, S. Demokritov, P. Grünberg, Phys. Status Solidi A 125, 635 (1991).

[9] W.H. Press, S.A. Teukolsky, W.T. Vetterling, B.P. Flannery, Numerical Recipes in C: The Art of Scientific Computing, 2nd ed., Cambridge University Press, Cambridge 1997, p. 288. 